\title{
Quasiparticle scattering in superconductors
}

\author{
Zdeněk Janů ${ }^{\mathrm{a}, \mathrm{b}, *, 1}$ František Soukup ${ }^{\mathrm{a}}$ Rudolf Tichý ${ }^{\mathrm{a}}$ Georgy Tsoi ${ }^{\mathrm{a}, \mathrm{c}}$ Jan Hadač ${ }^{\mathrm{d}}$ \\ Jana Vejpravová ${ }^{b}$ \\ anstitute of Physics AS CR, Na Slovance 2, 18221 Prague, Czech Republic \\ ${ }^{\mathrm{b}}$ Faculty of Mathematics and Physics, Charles University, Ke Karlovu 3, 12116 Prague \\ ${ }^{\mathrm{c}}$ Department of Physics and Astronomy, Wayne State University, Detroit, MI 48202, USA \\ ${ }^{\mathrm{d}}$ Faculty of Nuclear Sciences and Physical Engineering, Technical University of Prague, Břehová 7,11519 Prague
}

\begin{abstract}
We compare results of high-resolution magnetic flux (susceptibility) measurements in very weak magnetic field, performed of three classes of superconductors. They reveal astonishing details of the transition to the superconducting state. Although $\mathrm{Pb}$ behaves also on this scale according to BCS predictions, La is more reminiscent of HTS materials, which exhibit anomalous features. We suggest that known structure peculiarities are due to a strong electron-lattice instability and lead to a resonance electron back scattering.
\end{abstract}

Key words: diamagnetism, correlated electrons, electron-lattice instability, soft mode, fluctuations

The interaction that causes conventional superconductivity originates from a charge fluctuation with a frequency just below a characteristic frequency of the ionic lattice that excites in the lattice a resonant sympathetic vibration that overcompensates for the electronic charge. As a result, part of the interaction between two electrons in the medium is a spatially shortranged, temporally retarded attraction [1]. The drift current resulting from electron scattering, particularly its dependence on temperature $T$, the applied field $H$, and the frequency of $a c$ field $\omega$, may be observed detecting the flux generated by current induced in the sample by applied field. An elastic electron scattering gives rise to imaginary electrical conductivity while an inelastic one to real (Ohmic) conductivity.

The method used here allows to resolve variation of the "wave-vector" as low as $10^{3} \mathrm{~m}^{-1}$ and energy per electron in the order of $1 \mathrm{feV}$, which is six orders of magnitude better than give contact methods. In infinitesimally thin sample (wire oriented parallel with

\footnotetext{
* Corresponding Author: Email: janu@fzu.cz

1 This work was supported by the GACR (No. 102/02/0994) and by projects No. AVOZ1-010-914 and K1010104.
}

field), the magnetization produced by the shielding current is $\nabla \times \mathbf{M}=\mathbf{j}=(n e / m)(\hbar \mathbf{K}-e \mathbf{A})=i \omega \sigma \mathbf{A}$. The $\mathbf{K}(T)=\sum \mathbf{k} c_{k-K}^{*} c_{k}$ is the temperature dependent wave-vector of the paramagnetic quasiparticle counterflow current, and $\sigma(T)$ is electrical conductivity. Above $T_{c}$ the paramagnetic drift current cancels the diamagnetic one, $\mathbf{j}=-\left(n e^{2} / m\right) \mathbf{A}$, which is linear in response to applied field. A common interpretation of temperature dependence of conductivity (susceptibility) of superconductors is based on the temperature dependent superfluid density $n_{s}(T)$. However, it turns out that measurements in very low magnetic fields can be hardly interpreted this way. A more plausible concept is based on the temperature dependent quasi-particle scattering [2].

In conventional BCS superconductors the electronelectron pairing mechanism is mediated by low angle electron-phonon forward scattering. The superconducting-normal ( $\mathrm{SN}$ ) transition in type-I superconductors measured by $a c$ susceptibility (inductively) in weak magnetic fields is very sharp. (In $6 \mathrm{~N}$ pure long thin Ga single crystal it is $90 \%$ complete in a temperature interval of $2 \times 10^{-6} \mathrm{~K}$ [3].) Since near below $T_{c}$ the gap opens linearly with temperature, 
$\Delta(T) \approx \Delta^{\prime}\left(T_{c}\right)\left(T-T_{c}\right)$. The temperature dependent paramagnetic current follows the number of thermally excited quasi-particles, i.e., $\tanh \left(\Delta(T) / 2 k_{B} T\right)$. The data recorded on $\mathrm{Pb}$ sphere fit this dependence with the $\Delta^{\prime}\left(T_{c}\right) \approx 1.3 \mathrm{eV} / \mathrm{K}$, see Fig. 1 . The transition width is $\delta T \approx 1 \mathrm{mK}$, that gives the $\Delta(0)=$ $\Delta^{\prime}\left(T_{c}\right) \delta T \approx 1.3 \mathrm{meV}$, the value compatible with values $E_{g}(0)=2 \Delta(0)=2.73 \mathrm{meV}$ obtained by spectroscopy or specific heat measurement. In the case of $\mathrm{Pb}$ slab, the broadening of transition may be attributed to a demagnetization factor, which gives rise to non-homogeneous $\Delta^{\prime}(H, T)$.

La is commonly regarded as a type I superconductor. But its behaviour somewhat differs from the BCS model. La has two structure phases, fcc and hcp, with superconducting critical temperatures $6 \mathrm{~K}$ and 4.88 $\mathrm{K}$ respectively. An iso-structural phase transition, attributed to electronic and lattice instability, has an underlying dynamic mechanism of electronic origin. The electronic system is strongly coupled to lattice. The Fermi surface (FS) is modified at a general point at the zone boundary, thus involving a rather large degeneracy of inequivalent saddle points passing through $E_{F}$ [4]. A nesting feature of the FS is reduced at higher temperature [5]. This second-order phase transition could be described by a zone-boundary soft phonon mode, that may ultimately result in the static distortion. The modulated phase is, in general, an incommensurate one, which, may eventually lock into a commensurate modulation due to the freezing of a specific phonon and result in higher-order (anharmonic) terms in the strain energy [6]. Comparing to $\mathrm{Pb}$, the temperature dependence of imaginary part of $a c$ conductivity of La shows: i) linear segments; ii) low temperature part fitting Lorentzian, $\operatorname{Im} \sigma(T) \propto 1 /\left(\left(T-T_{c}\right)^{2}+\Gamma^{2}\right)$; iii) at decreasing $H_{a c}$ the dips rise below $T_{c}$. They increase in amplitude, shift toward $T_{c}$, and are accompanied by "noise" in real part. These features are extrinsic to the vortex matter, to BCS or Ginzburg-landau models, but they can be related to displacive structural transition and modulated phases.

In HTS cuprates, the temperature dependence of conductivity is much more reminiscent of La than of $\mathrm{Pb}$. The $\operatorname{Im} \sigma(T)$ may be well approximated by $\operatorname{Im} \sigma(T) \propto \arctan \left(2\left(T-T_{c}\right) / \Gamma\right)$, the Breit-Wigner resonance [7]. The $S$-shaped form with symmetry around $T_{c}$ and linear parts are inconsistent with the fluctuation model, which is widely used to explain the convex part. With increasing $H_{a c}$ the absorption peak on $\operatorname{Re} \sigma(T)$ broadens and shifts toward the lower temperature whereas the opposite is expected. The sharpness of the transition $\left(10 \mathrm{mK}\right.$ at $\left.T_{c}=91 \mathrm{~K}\right)$ suggests high underlying energies. We propose that this data may be understood as resonance electron (back)scattering from modulated lattice. Such a view is consistent with known data of the FS properties and lattice structure

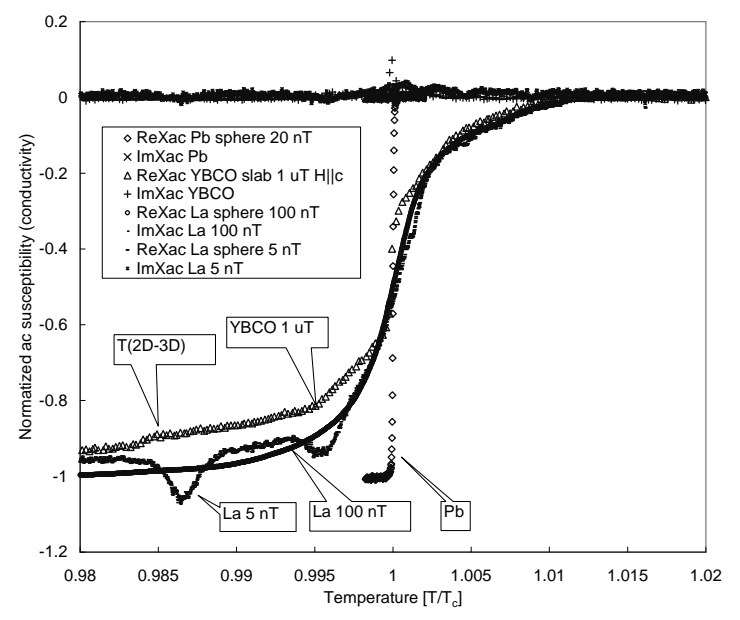

Fig. 1. The $\mathrm{SN}$ transitions in $\mathrm{Pb}$ and $\mathrm{La}$ spheres and $\mathrm{YBCO}$ single-crystal slab oriented $c \| \mathbf{H}_{a c}$. The transition in $\mathrm{Pb}$ falls in known data [8]. In YBCO transition occurs from $2 \mathrm{D}$ to $3 \mathrm{D}$ superconductivity at temperature marked $T(2 D-3 D)$ [13].

available by other experimental methods, which show strong electron localization, stripes, CDW, SDW, pseudo-gap, real-space gap, and other peculiarities $[9,10,11,12]$.

We believe that because of the similar behavior of La and HTS, but different from the character of $\mathrm{Pb}$, the insight into the diamagnetism of conduction electrons in "simple" (as compared with HTS) La with its structure peculiarities, is relevant for understanding of a mechanism of superconductivity in HTS. In particular, we conjecture the relation between the correlated electrons (pairing) and electron-lattice instability, and between the elastic electron scattering and the soft mode or static lattice distortion and strain.

Authors are grateful to L. Havela for stimulating discussion and comments.

\section{References}

[1] V. Ambegaokar, in Superconductivity, Ed. By R.D. Parks, M. Dekker Inc. NY, 1969, Vol. 1, p. 260

[2] J. Bardeen et al., Phys. Rev. 108 (1957) 1175.

[3] W.D. Gregory et al., Phys. Rev. 165 (1968) 556.

[4] W.E. Pickett et al., Phys. Rev. B 22 (1980) 2695.

[5] X.W. Wang et al., Phys. Rev. B 33 (1986) 3851.

[6] T. Castan et al., Phys. Rev. B 67 (2003) 134113.

[7] Z. Janu et al., Europhysics Letters 64 (2003) 399.

[8] J.W. Schooley et al., Adv. Cryog. Eng. 17 (1972) 192.

[9] T. Sato et al., Phys. Rev. Lett. 89 (2002) 067005-1.

[10] M. Kugler et al., Phys. Rev. Lett. 86 (2001) 4911.

[11] Y. Zhu et al., Phys. Rev. Lett. 76 (1996) 443. 
[12] S.A. Kivelson et al., Rev. Mod. Phys. 75 (2003) 1201.

[13] Z. Janu et al., Physica C 388 (2003) 751. 